\title{
Abscesos piógenos encefálicos. Nuestra experiencia en 60 casos consecutivos
}

M. Ortega-Martínez; J.M. Cabezudo; I. Fernández-Portales; L. Gómez-Perals; J.A. Rodríguez-Sánchez; L. GarcíaYagüe; L.F. Porras; L.M. Lorenzana; F. Ugarriza; M. Pineda-Palomo y L.M. Bernal-García

Servicio de Neurocirugía. Hospital Regional Universitario Infanta Cristina. Badajoz

Resumen

Introducción. El absceso cerebral es un proceso supurativo focal en el parénquima cerebral que todavía presenta una elevada mortalidad. El resultado está altamente relacionado con un tratamiento precoz y adecuado. Para valorar dicho tratamiento revisamos los abscesos cerebrales tratados en nuestro Servicio en los últimos 14 años

Material y métodos. Presentamos una revisión de los 60 pacientes con diagnóstico de absceso cerebral piógeno tratados en el Servicio de Neurocirugía del Hospital Infanta Cristina entre Enero de 1990 y Febrero de 2004, prestando atención a la epidemiología, clínica, etiología, microbiología, tratamiento y resultado final.

Resultados. La relación hombre/mujer es 5.6/1, con una edad media de 47 años; el origen de la infección más frecuente es el hematógeno, seguido de las infecciones contiguas, con un $\mathbf{2 2 \%}$ de abscesos de origen desconocido; los gérmenes más frecuentes son los cocos Gram positivos ( $44 \%)$, con una importante presencia de gérmenes anaerobios (40\%); en un $39 \%$ de los casos las infecciones son mixtas; las modalidades de tratamiento fueron la punción de los abscesos para aspiración-drenaje, la cirugía abierta, con o sin resección capsular, y el tratamiento médico exclusivo. 52 pacientes $(86.7 \%)$ curaron sin secuelas y $4(6,7 \%)$ fallecieron. Aunque los resultados de ambos tipos de tratamiento quirúrgico son similares, el drenaje del absceso requirió en un $20 \%$ de los casos un segundo proceder quirúrgico, mientras que la craneotomía lo precisó sólo en el $10 \%$; la estancia media en nuestro hospital fue mucho menor en los abscesos tratados mediante drenaje que en los tratados mediante cirugía abierta (13 vs 26 días); la mortalidad fue mayor en pacientes con bajo nivel de conciencia al ingreso y en mayores de $\mathbf{7 0}$ años.

Conclusiones. Consideramos que la menor estancia media del drenaje del absceso, unido a su alta eficacia y su baja morbilidad, hacen aconsejable su uso como tratamiento de primera elección.

Recibido: 07-01-05. Aceptado: 04-03-05
PALABRAS CLAVE: Abscesos cerebrales. Escisión. Infección intracraneal. Punción-aspiración.

Pyogenic brain abscesses: experience with 60 consecutive cases

Summary

Introduction. Brain abscess is a focal suppurative process in the brain parenchyma that still carries high mortality rates. Outcome is closely related with a correct and early management. In order to evaluate this management we have reviewed the brain abscesses treated in our Department during the last 14 years.

Material and Methods. The authors present a retrospective series of 60 consecutive patients with pyogenic brain abscess treated between January of 1990 and February of 2004 paying attention to the epidemiology, etiology, clinical data, microbiology, treatment modalities and outcome

Results. The male to female rate was 5.6 to 1 . The average age was 47 years. Hematogenous spread was most frequent, followed by contiguous spread. In $22 \%$ of the cases, the origin was unknown. Regarding the causative pathogens, Gram positive cocci are the most frequent (44\%), with a $40 \%$ incidence of anaerobics. A mixed infection occurred in $39 \%$ of the abscesses. Three modalities of treatment were used: non surgical, catheter drainage-aspiration and surgical excision. Outcome was excellent in 52 patients $(86.7 \%)$ and 4 patients $(6.7 \%)$ died. Although outcome was similar in both surgical modalities, drainage-aspiration required a second procedure in $20 \%$ of the cases while this was necessary in only $10 \%$ of the patients with abscess excision. Length of admission was shorter in the drainageaspiration group than in the excision group (13 and 26

Abreviaturas. BGN: bacilos Gram negativos. GOS: Glasgow Outcome Scale. LCR: líquido cefalorraquideo. SNC: sistema nervioso central. TC: tomografía computarizada. VSG: velocidad de sedimentación globular. 
days respectively). Mortality was higher in patients with low level of consciousness and age over 70 years.

Conclusions. The shorter admission time associated with drainage-aspiration of brain abscesses together with its high efficacy and low morbidity suggests that drainageaspiration should be used as the first mode of treatment.

KEY WORDS: Brain abscess. Drainage-aspiration. Excision. Intracranial infection.

\section{Introducción}

El absceso cerebral es un proceso supurativo focal en el parénquima cerebral que complica sinusitis paranasales, otitis medias, cirugía, traumatismos o bacteriemias provenientes de focos extracraneales. Su patogénesis, clínica, tratamiento y pronóstico fue magistralmente descrito por Sir William Macewen en 1893 en su monográfico sobre infecciones del sistema nervioso central (SNC) ${ }^{13}$. Macewen tuvo unos resultados excepcionalmente buenos en los abscesos que operó, con una mortalidad del 5\%, lo que le llevó a concluir que en el absceso cerebral no complicado, operado a tiempo, la recuperación debe ser la regla ${ }^{13}$. Pese a ello, las tasas de mortalidad registradas en los años 70 llegaban hasta el $50 \%{ }^{16}$. Desde entonces, gracias fundamentalmente al diagnóstico precoz con la TC cerebral, estas tasas de mortalidad han descendido hasta situarse en los 90 entre el 5-10\% $\%^{5,15,28}$. Todavía, no obstante, siguen siendo una patología grave, que requiere alto nivel de sospecha y una actuación terapéutica rápida. Entre las opciones de tratamiento se encuentran la craneotomía para extirpación del absceso, la punción del mismo para aspiración del contenido purulento, o el tratamiento exclusivamente médico. En vistas a valorar los resultados del tratamiento en los casos atendidos por nosotros, revisamos los pacientes con abscesos cerebrales que hemos atendido en nuestro hospital a lo largo de 14 años.

\section{Pacientes y método}

El estudio describe 60 pacientes con abscesos cerebrales piógenos atendidos por el Servicio de Neurocirugía del Hospital Universitario Infanta Cristina de Badajoz, a lo largo de 14 años (desde Enero de 1990 hasta Febrero de 2004), todos con un seguimiento mínimo de 6 meses. Los datos clínicos, radiológicos y de laboratorio fueron recogidos retrospectivamente a través de las notas de nuestros informes y ficheros y a través de la revisión de las historias clínicas de los pacientes. La evolución posterior del paciente, a través de las notas de las revisiones hasta que fuera dado de alta definitiva.

Todos los pacientes tienen confirmación anatomopatológica o microbiológica del proceso infeccioso, excepto los casos en que se siguió tratamiento médico exclusivo sin toma de biopsia. Estos fueron incluidos en el estudio en función de sus características clínico-radiológicas y de su evolución ante el tratamiento antibiótico administrado.

Las opciones de tratamiento iniciales fueron tres: 1. Punción para aspiración-drenaje del absceso, a mano alzada o guiado con estereotaxia; 2. Cirugía abierta, con exéresis completa, que incluye la cápsula del absceso, o exéresis parcial, es decir, la cápsula permanece in situ; y 3.

\section{Tratamiento conservador.}

Los datos recogidos son los siguientes: edad y sexo, estancias hospitalarias, antecedentes personales, clínica, datos de laboratorio (sangre y LCR), localización, tamaño y número de los abscesos, microbiología, tratamiento y resultados del mismo.

Los resultados del tratamiento se han clasificado según la Glasgow Outcome Scale (GOS), tomando como definitivo el estado del paciente a los 6 meses del diagnóstico o al alta si esta se ha producido antes. La mortalidad se ha definido como cualquier fallecimiento durante la estancia hospitalaria o en los 30 días posteriores al alta. Los casos en que ha habido más de una opción de tratamiento en un paciente, o más de una actividad quirúrgica, se agrupan bajo el epígrafe Reintervenciones. En este grupo hay un subgrupo de pacientes que requirieron pasar a una opción de tratamiento más agresiva. Se incluyen bajo el epígrafe Grupo de cambio de tratamiento.

\section{Resultados del estudio}

La edad media de nuestros pacientes es de 47 años (rango 11-79 años), con una clara preponderancia en varones y mayor incidencia en la $7^{\text {a }}$ década (Tabla 1$)$.

\section{Tabla I}

Distribución de pacientes por edad y sexo y mortalidad según estos factores $(n=60)$

\begin{tabular}{l|ccc}
\hline Sexo & $N^{o}$ pacientes & Porcentaje & Exitus \\
\hline Hombres & 51 & 85 & 4 \\
Mujeres & 9 & 15 & 0 \\
Edad & & & \\
$0-10$ & 0 & 0 & \\
$11-20$ & 5 & 8.3 & \\
$21-30$ & 10 & 16.7 & \\
$31-40$ & 7 & 11.7 & 1 \\
$41-50$ & 11 & 18.3 & \\
$51-60$ & 8 & 13.3 & \\
$61-70$ & 12 & 20 & \\
$>71$ & 7 & 11.7 & 3 \\
\hline
\end{tabular}


Tabla 2

Datos clínicos y de laboratorio

\begin{tabular}{l|cc}
\hline Signos y Síntomas & $N^{\circ}$ pacientes & Porcentaje \\
\hline Focalidad neurológica & 38 & 63.3 \\
Cefalea +/- síntomas de HIC & 36 & 60 \\
Síndrome febril & 31 & 51.7 \\
Deterioro funciones superiores, bradipsiquia, agitación & 16 & 26.7 \\
Crisis epilépticas & 10 & 16.7 \\
Signos meníngeos & 7 & 11.7 \\
& & \\
GCS & & 45 \\
$\quad 15$ & 27 & 28.3 \\
$\quad 13-14$ & 17 & 18.3 \\
$\quad<-12$ & 11 & 8.3 \\
$\quad 9$ & 5 & 41.7 \\
Leucocitos $<11000$ leu/mm ${ }^{3}$ & & 38.3 \\
$\quad 11001-16000$ leu/mm & & 20 \\
$\quad>16000$ leu/mm & & \\
Neutrofilia (más de $74 \%$ de neutrófilos en la fórmula leucocitaria) & 25 & 65 \\
LCR patológico (hiperproteinorraquia, hipoglucorraquia o pleocitosis) & 23 & 56.3 \\
\hline
\end{tabular}

HIC; hipertensión intracraneal. LCR: liquido cefalorraquideo. GCS: Escala de Coma de Glasgow.

En la clínica domina la focalidad neurológica, seguida de la cefalea, el síndrome febril y la alteración del nivel de conciencia, en la mitad de los casos de carácter leve. La leucocitosis y la neutrofilia sólo se dan en 58 y $65 \%$ de los casos respectivamente. El LCR se investigó en 16 ocasiones, resultando patológico en 9 pacientes (Tabla 2).

Los abscesos más frecuentes en nuestra serie son los hematógenos o metastásicos de una infección a distancia, seguidos de los abscesos que tienen su origen en una infección contigua. En un $22 \%$ de los casos, no conocemos la procedencia de la infección (Tabla 3).

El mayor número de abscesos únicos está en los lóbulos frontal y temporal, ambos con el mismo número de abscesos. El 13\% tenían abscesos múltiples, la mayoría provenientes de infecciones metastásicas (vía hematógena) o de los abscesos de origen desconocido (Tabla 3). La localización de los abscesos está muy relacionada con el origen de la infección, como se muestra en la tabla 4.

Se han aislado 54 gérmenes diferentes, en 38 abscesos. En los 22 casos restantes no se llegó a conocer el germen causante de la infección. En 15 casos la infección era mixta. Los gérmenes más frecuentes fueron los cocos Gram positivos areobios, seguido de los gérmenes anaerobios. Los bacilos Gram negativos (BGN), poco frecuentes, se encon- traban fundamentalmente en abscesos relacionados con infecciones otógenas. En los abscesos hematógenos y los de origen desconocido hay alto porcentaje de abscesos con cultivos negativos ( 40 y $46 \%$ respectivamente) y cuando se aislan gérmenes predominan los cocos Gram positivos aerobios. En los abscesos relacionados con cirugía o traumatismos destaca la presencia de anaerobios, junto a los clásicos cocos Gram + aerobios (Tabla 5).

Las modalidades de tratamiento pueden resumirse en tres: punción para aspiración-drenaje, cirugía abierta y tratamiento conservador (Tabla 6).

En líneas generales, la primera opción terapéutica ha sido la punción del absceso, bien a mano alzada, bien estereotáctica, aspirando el contenido purulento y dejando en la cavidad un catéter para drenaje continuo durante 24-48 horas. Este tratamiento se realizó en 35 pacientes (58\%).

La craneotomía, con o sin resección de la cápsula del absceso, se ha realizado en 20 pacientes $(33 \%)$. Se ha optado por la cirugía abierta como primera opción en los abscesos cerebelosos debido a las dificultades de aspiración, a la frecuente hidrocefalia obstructiva que provocaban y al riesgo de deterioro súbito; en los abscesos postraumáticos, dada la frecuencia de cuerpos extraños retenidos en estos casos; 
Tabla 3

Origen, localización y tamaño de los abscesos $(n=60)$. Se señala también el porcentaje de abscesos múltiples entre el total de pacientes y su etiología

\begin{tabular}{|c|c|c|c|}
\hline Origen & $N^{\circ}$ pacientes & Porcentaje & Exitus \\
\hline Hematógeno & 22 & 36,7 & \\
\hline Pulmonar & 9 & & \\
\hline Endocarditis & 5 & & \\
\hline Cardiopatías sin endocarditis & 2 & & \\
\hline Abscesos dentarios & 3 & & \\
\hline Inmunodepresión & 3 & & 1 \\
\hline Infección en contigüidad & 18 & 30 & \\
\hline Oídos/Mastoides & 14 & & 2 \\
\hline Senos paranasales & 4 & & \\
\hline Postquirúrgicos / Postraumáticos & 7 & 11.7 & 1 \\
\hline Desconocido & 13 & 21.7 & \\
\hline \multicolumn{4}{|l|}{ Localización } \\
\hline Temporal & 16 & 26.7 & 1 \\
\hline Frontal & 16 & 26.7 & 2 \\
\hline Cerebelo & 7 & 11.7 & 1 \\
\hline Parietal & 6 & 10 & \\
\hline Occipital & 3 & 5 & \\
\hline Tálamo y ganglios basales & 2 & 3.3 & \\
\hline Tronco y IV ventrículo & 2 & 3.3 & \\
\hline Múltiples & 8 & 13.3 & \\
\hline \multicolumn{4}{|l|}{ Tamaño } \\
\hline$\leq 2.5 \mathrm{~cm}$ de diámetro & 19 & 31.7 & 2 \\
\hline $2.6-4.9 \mathrm{~cm}$ de diámetro & 19 & 31.7 & 1 \\
\hline$\geq 5 \mathrm{~cm}$ de diámetro & 22 & 36.7 & 1 \\
\hline Abscesos múltiples $(n=8)$ & 8 & & 0 \\
\hline Hematógenos & 3 & 37.5 & \\
\hline Desconocidos & 3 & 37.5 & \\
\hline Otógenos & 1 & 12.5 & \\
\hline Postquirúrgicos & 1 & 12.5 & \\
\hline Sinusales & 0 & 0 & \\
\hline
\end{tabular}

en algunos abscesos localizados en la porción anterior del lóbulo temporal, que se extirparon en bloque mediante una lobectomía parcial; en aquellos casos que asemejaban ser un tumor; y, finalmente, en los casos en que existía un deterioro rápido o profundo del paciente. Las preferencias del cirujano y su experiencia con uno u otro tipo de trata- miento, también han tenido influencia a la hora de escoger entre un drenaje y una cirugía abierta.

El tratamiento conservador se realizó en 5 ocasiones (8.3\%): en abscesos de pacientes inmunodeprimidos sugestivos de toxoplasmosis; en abscesos de difícil abordaje quirúrgico, como el absceso de tronco; y en alguno de los 
Tabla 4

Relación de la localización de los abscesos con el origen de los mismos

\begin{tabular}{|c|c|c|c|c|c|c|c|c|c|}
\hline Origen & Frontal & Temporal & Parietal & Occipital & Cerebelo & $\begin{array}{l}\text { Ganglios } \\
\text { basales }\end{array}$ & Tronco & Múltiples & TOTAL \\
\hline Oídos & & $8(57 \%)$ & & & $5(36 \%)$ & & & $1(7 \%)$ & 14 \\
\hline $\begin{array}{l}\text { Senos } \\
\text { paranasales }\end{array}$ & $4(100 \%)$ & & & & & & & & 4 \\
\hline Hematóg. & $8(36 \%)$ & $4(18 \%)$ & $2(9 \%)$ & $2(9 \%)$ & & $1(4 \%)$ & $2(9 \%)$ & $3(14 \%)$ & 22 \\
\hline Desconoc. & $1(8 \%)$ & $2(15 \%)$ & $3(23 \%)$ & $1(8 \%)$ & $2(15 \%)$ & $1(8 \%)$ & & $3(23 \%)$ & 13 \\
\hline $\begin{array}{l}\text { Postraumát/ } \\
\text { Postquir. }\end{array}$ & $3(43 \%)$ & $2(29 \%)$ & $1(14 \%)$ & & & & & $1(14 \%)$ & 7 \\
\hline TOTAL & 16 & 16 & 6 & 3 & 7 & 2 & 2 & 8 & 60 \\
\hline
\end{tabular}

Tabla 5

Resultados de los cultivos. En las infecciones mixtas cada gérmen se ha contabilizado de forma independiente. Se muestra también la microbiología en relación con la etiología

\begin{tabular}{l|cccccc}
\hline Microorganismos & $N^{\circ}$ abscesos & Oído & Senos & $\begin{array}{c}\text { Postraum. } \\
\text { Postquirur. }\end{array}$ & Hematóg. & Desconoc. \\
\hline Cocos Gram + & $24 / 54(44.4 \%)$ & 4 & 3 & 2 & 9 & 6 \\
Bacilos Gram + & $2 / 54(3.7 \%)$ & 0 & 0 & 0 & 1 & 1 \\
Bacilos Gram - & $6 / 54(11.1 \%)$ & 5 & 0 & 1 & 0 & 0 \\
Anaerobios & $22 / 54(40.7 \%)$ & 8 & 3 & 2 & 6 & 3 \\
Cultivos negativos & 22 & 4 & 0 & 3 & 9 & 6 \\
Infección polimicrobiana & 15 & 5 & 3 & 1 & 3 & 3 \\
\hline
\end{tabular}

Tabla 6

Modalidades de tratamiento realizado en primera opción y casos que requirieron reintervención

\begin{tabular}{c|cc}
\hline Tratamiento & En primera opción & Reintervención \\
\hline Aspiración-Drenaje & $35(58.3 \%)$ & $7(20 \%)$ \\
A mano alzada & 23 & \\
Estereotáctico & 12 & $2(10 \%)$ \\
Exaneotomla & $20(33.3 \%)$ & \\
Exéresis parcial & 12 & $4(80 \%)$ \\
Tratamiento conservador & $5(8.3 \%)$ & 13 \\
\hline
\end{tabular}

pacientes con abscesos pequeños, múltiples, y/o patología médica importante que desaconsejaban la intervención quirúrgica.

Aunque las modalidades de tratamiento escogidas en primera opción fueron las reseñadas en los párrafos anteriores, en 13 pacientes (22\%) hubo de realizarse una segunda intervención quirúrgica tras fracaso de la primera (por recidivas, por fracaso en la técnica, o por mala evolución en el tratamiento conservador). Se recogen bajo el epígrafe Reintervenciones. En este grupo hay un subgrupo de 7 pacientes en que se precisó cambiar el tratamiento inicial por otra opción más agresiva (de tratamiento conservador 
Tabla 7

Detalle de los pacientes que requirieron reintervenciones. El porcentaje (entre paréntesis) va en relación al método de tratamiento utilizado en primera opción

\begin{tabular}{|c|c|c|c|c|}
\hline Tratamiento & Aspiración-Drenaje & Craneotomía & Tto Conservador & TOTAL \\
\hline Reintervención & $7(20 \%)$ & $2(10 \%)$ & $4(80 \%)$ & $13(21.7 \%)$ \\
\hline Mismo tratamiento & & & & $6(46 \%)$ \\
\hline Drenaje $\rightarrow$ Drenaje & 4 & & & \\
\hline Craneotomía $\rightarrow$ Craneotomia & & 2 & & \\
\hline Cambio de tratamiento & & & & $7(54 \%)$ \\
\hline Drenaje $\rightarrow$ Craneotomía & 3 & & & \\
\hline Tto conservador $\rightarrow$ Drenaje & & & 4 & \\
\hline
\end{tabular}

Tabla 8

Detalle de los resultados globales y según tratamiento, asi como de las estancias hospitalarias en pacientes que recibieron tratamiento quirúrgico. Las estancias se catalogan en función del tratamiento que se escogió en primera opción.

Los pacientes del Grupo de cambio de tratamiento se valoran aparte, en cuanto a sus resultados y las estancias

\begin{tabular}{l|cllll}
\hline Tratamiento & $\begin{array}{l}\text { GOS } 5 \\
\text { Buena } \\
\text { recuperación }\end{array}$ & $\begin{array}{l}\text { GOS } 4 \\
\text { Incapacidad } \\
\text { moderada }\end{array}$ & $\begin{array}{l}\text { GOS 3 } \\
\text { Incapacidad } \\
\text { severa }\end{array}$ & $\begin{array}{l}\text { GOS 1 } \\
\text { Exitus }\end{array}$ & $\begin{array}{l}\text { Estancias } \\
\text { medias }\end{array}$ \\
\hline $\begin{array}{l}\text { Aspiración-Drenaje } \\
\text { Craneotomía }\end{array}$ & $\begin{array}{l}29(90.6 \%) \\
\text { Tratamiento conservador }\end{array}$ & $17(3.1 \%)$ & $1(3.1 \%)$ & $1(3.1 \%)$ & $13.5($ rango 1-34) \\
Cambio de tratamiento & $1(100 \%)$ & & $1(5 \%)$ & $2(10 \%)$ & $26.5(7-112)$ \\
TOTAL & $5(71.4 \%)$ & $1(14.3 \%)$ & & $1(14.3 \%)$ & $8.7(1-17)$ \\
& $52(86.7 \%)$ & $2(3.3 \%)$ & $2(3.3 \%)$ & $4(6.7 \%)$ & $17.1(1-112)$ \\
\hline
\end{tabular}

a drenaje, o de drenaje a craneotomía). Son el denominado Grupo de cambio de tratamiento: 4 de estos pacientes fueron inicialmente tratados de forma conservadora $y$ posteriormente se realizó drenaje de los abscesos, y otros 3 fueron abscesos inicialmente drenados y posteriormente tratados mediante cirugía abierta (Tabla 7).

Todos los pacientes, independientemente de la modalidad de tratamiento médico o quirúrgico administrado, recibieron tratamiento antibiótico empírico, según el protocolo del Servicio, con vancomicina ( 1 gr cada 12 horas), ceftazidima (2 gr cada 8 horas) y metronidazol (500 mg cada 8 horas), por vía intravenosa. Esta pauta de antibioterapia fue supervisada en todos los casos por el Servicio de Enfermedades Infecciosas y ajustada posteriormente en relación al antibiograma. Siempre que ha sido posible, tras las primeras semanas de antibioterapia intravenosa se completó el tratamiento con antibióticos por vía oral, hasta completar 6 semanas de tratamiento. Además, en todos los pacientes se realizó profilaxis anticomicial con fenitoína. En un $75 \%$ de los pacientes se usaron esteroides.

En el conjunto de toda la serie se han producido 4 fallecimientos (GOS 1), 2 casos de incapacidad moderada (GOS 4) y 2 casos de incapacidad grave (GOS 3). En 52 de los 60 casos (86.7\%) el resultado fue óptimo (GOS 5) (Tabla 8)

La punción-drenaje del absceso se realizó en primera opción en 35 pacientes y, dejando aparte los casos en que se tuvo que cambiar el tratamiento, tuvo un resultado óptimo en el $91 \%$ de los casos, aunque en el $20 \%$ de los pacientes se requirió una segunda intervención. La craneotomía, que tuvo unos resultados óptimos en el $85 \%$ de los casos, sólo precisó reintervención en 2 de los 20 pacientes (10\%). Todos los pacientes que recibieron exclusivamente tratamiento médico tuvieron un resultado final excelente, aunque en 4 de los 5 se precisó puncionar el absceso debido a una mala evolución clínica.

Los pacientes del Grupo de Cambio de Tratamiento constituyen un grupo heterogéneo en el que se incluyen los 
4 pacientes en que se pasó del tratamiento médico exclusivo a la punción ( $100 \%$ de GOS 5$)$ y los 3 pacientes en que se pasó de punción a craneotomía (1 éxitus, 1 caso de incapacidad moderada, y 1 caso con resultado óptimo). En total esto corresponde a un $71.4 \%$ de resultados óptimos para este grupo.

En relación con el uso de esteroides, no hemos encontrado diferencias apreciables en los resultados finales.

Las estancias hospitalarias varían entre 1 y 112 días, con una estancia media de 17.1 días (Tabla 8). Los pacientes en que se realizó una cirugía abierta tuvieron una estancia media mucho mayor que aquellos en que se realizó punción para drenaje del absceso (26 vs 13 días). En esta estadística se considera a los pacientes en relación al tratamiento elegido en primera opción, dejando aparte los pacientes en los que el tratamiento se modificó, que tuvieron una estancia media de 8,7 días.

De los 4 pacientes fallecidos, uno de ellos llegó a nuestro Servicio en muy mal estado clínico, con GCS de 4 puntos; otro era un paciente inmunodeprimido por corticoterapia crónica que desarrolló un absceso por listerias; el tercero falleció por complicaciones médicas tras la extirpación de un absceso multiloculado en fosa posterior, de origen otógeno y flora mixta; y por último, el cuarto paciente desarrolló un absceso postquirúrgico a germen desconocido que evolucionó de forma desfavorable pese al tratamiento quirúrgico y antibioterapia empírica.

Hemos encontrado una mayor mortalidad en pacientes con baja puntuación de Glasgow al ingreso y con edad avanzada: la mortalidad entre los pacientes con GCS menor o igual a 12 al ingreso fue de $12.5 \%$ vs. un $4.3 \%$ de mortalidad para los pacientes con GCS $>12$. Con respecto a la edad, 3 de los 4 pacientes fallecidos eran mayores de 70 años. En relación al sexo, todos los fallecidos eran varones. No hay diferencias apreciables respecto al tamaño de los abscesos o la multiplicidad de los mismos.

\section{Discusión}

El absceso cerebral se inicia cuando los gérmenes alcanzan el parénquima cerebral, lo cual se produce a través de tres vías fundamentales: por inoculación directa o fístulas, en el caso de traumatismos o cirugía; por una infección en contigüidad (bien por invasión directa, bien por tromboflebitis de las venas emisarias); o por embolismos sépticos procedentes de infecciones a distancia. Tradicionalmente las infecciones crónicas del oído y de los senos paranasales han sido la causa más frecuente de abscesos cerebrales ${ }^{11,16}$, pero su incidencia ha disminuido con la mejora del tratamiento de estas infecciones, produciéndose así un relativo incremento de la infección de origen hematógeno ${ }^{28}$, que en nuestra serie es la que ocupa el primer lugar. El origen se desconoce en un porcentaje de casos que varía del 10 al
$40 \%{ }^{11,16,28}$. En nuestra serie estos abscesos criptogenéticos son un $22 \%$.

El absceso cerebral es una patología que se da entre personas jóvenes o de edad media (entre la segunda y cuarta décadas de la vida) ${ }^{6,11,16}$, con una inexplicable predilección por el sexo masculino. En nuestra serie la relación hombre/ mujer es de 5.6/1 y la edad media de 47 años.

Aunque no hay datos patognomónicos que indiquen la existencia de supuración intracraneal, comportándose un absceso cerebral del mismo modo que un tumor, es muy sugerente la asociación de cefalea, disminución del nivel de conciencia, focalidad neurológica y síndrome febril, especialmente si presenta una evolución rápida de los síntomas. Entre el 70 y el $97 \%$ de los pacientes tienen cefalea, que suele ser progresiva y refractaria al tratamiento habitual, y aparece bien aislada, bien asociada a síntomas de hipertensión intracraneal ${ }^{1,5,6,11,17,28}$. En nuestra serie se observa en las dos terceras partes de los pacientes. La focalidad neurológica se detecta en la literatura en un $60 \%$ de los casos ${ }^{11}$. Nosotros hemos detectado alteraciones focales en un $64.5 \%$ de nuestros pacientes, constituyendo el primer complejo sintomático de presentación en frecuencia. La disminución del nivel de conciencia se da hasta en un $66 \%$ de los $\operatorname{casos}^{11}$. Entre nuestros pacientes la encontramos en un $53 \%$, siendo de carácter leve (somnolencia, desorientación) en un 50\%, manteniendo una puntuación de Glasgow de 13-14, mientras que el otro 50\% tiene 12 puntos o menos en dicha escala. La fiebre, que aparece aproximadamente en la mitad de los casos, suele ser de bajo grado, de modo que una temperatura muy elevada debe hacer sospechar una infección sistémica o meningitis concomitante ${ }^{11}$. Son también frecuentes en las distintas series las crisis epilépticas, que antes de la cirugía se dan entre un $25-50 \%^{11,16,17,22}$, aunque nosotros hemos recogido menor incidencia (17\%). Otros síntomas menos frecuentes son el meningismo, la ataxia de la marcha, etc.

En el diagnóstico es indiscutible el protagonismo que tiene la TC craneal, gracias a la cual, al permitir un diagnóstico precoz, ha mejorado sustancialmente el pronóstico de los abscesos cerebrales, disminuyendo la mortalidad en los mismos, actualmente por debajo del $10 \%$. Es, además, el método de elección usado para valorar la evolución del paciente. La RM cerebral ayuda mejor que la TC a detectar los abscesos múltiples y a realizar un diagnóstico de presunción, en casos de dudas diagnósticas. Por su parte, los hemogramas que hemos obtenido confirman su relativa utilidad para el diagnóstico del absceso cerebral: sólo había leucocitosis y neutrofilia en un 58 y $65 \%$ de los casos respectivamente. La velocidad de sedimentación globular (VSG) y la proteína C reactiva son parámetros más sensibles para valorar la evolución de la infección ${ }^{11}$, pero en nuestro Servicio no se han obtenido de forma rutinaria. Respecto al análisis del LCR, en 7 de las 16 ocasiones en 
que se ha investigado ha sido normal; su bajo rendimiento, unido al $15-33 \%$ de riesgo de deterioro neurológico recogido en la literatura, hace desaconsejable su estudio de manera rutinaria ${ }^{6,11,16,17}$.

La distribución de los abscesos cerebrales en nuestra serie es similar a la obtenida en otras, con predominio de los abscesos frontales y temporales. La localización de los abscesos está muy relacionada con la etiología de la infección, confirmándose en nuestra serie hechos repetidamente observados, como la predilección de los abscesos de origen otógeno por el lóbulo temporal o cerebelo, los de senos paranasales por el lóbulo frontal, o la frecuente multiplicidad de los de origen hematógeno.

La mejora de las técnicas microbiológicas ha hecho descender el número de abscesos con cultivo estéril. Si anteriormente esta tasa estaba en torno al $50 \%$, actualmente se encuentra entre el 25 al $30 \%$ en series seleccionadas ${ }^{11}$. No obstante, se han publicado series con cultivos positivos del $100 \%$, incluso con toma previa de antibióticos, gracias al uso de técnicas microbiológicas selectas ${ }^{12}$. En cuanto a los gérmenes responsables, en los últimos años hay una tendencia a aumentar los abscesos por BGN, por anaerobios, y por infecciones polimicrobianas ${ }^{11}$. Entre los nuestros, los gérmenes más frecuentes continúan siendo los cocos Gram positivos aerobios, especialmente los estreptococos del grupo viridans y los estafilococos spp, seguidos muy de cerca por los gérmenes anaerobios. Respecto a la microbiología, hay que tener en cuenta que lo más interesante es la relación entre la etiología de la infección y la edad del paciente, por un lado, y el germen productor, por otro, ya que permite la utilización empírica de antibióticos. En este sentido, en nuestra serie lo más destacado es la presencia de los BGN casi exclusivamente entre los abscesos de origen ótico, así como la presencia de gérmenes anaerobios en abscesos postcirugía o trauma.

Tres han sido las opciones de tratamiento empleadas por nosotros: el tratamiento conservador, la punción para aspiración-drenaje y la cirugía abierta, con o sin resección capsular. Heinemann y cols. fueron los primeros en sugerir que los abscesos cerebrales podían ser tratados con antibióticos exclusivamente ${ }^{8}$. Sus trabajos y otros posteriores fueron revisados por Rosenblum y cols. quienes llegaron a la conclusión de que dichos abscesos estaban la mayoría en fase de cerebritis (lo que facilita la llegada del antibiótico) y los gérmenes responsables en numerosos casos se habían obtenido de cultivos positivos de sangre, LCR o del sitio remoto de la infección ${ }^{23}$. En trabajos posteriores $^{24}$, se demostró que los abscesos en fase encapsulada podían también responder a antibióticos exclusivamente, si bien debían ser abscesos de un tamaño pequeño (2.1 $\mathrm{cm}$ de media). Sobre la base de dichos trabajos se acepta el tratamiento médico exclusivo para abscesos profundos de difícil acceso, como los de tronco, los abscesos de pequeño tamaño, y aquéllos que se encuentren en fase de cerebritis. Los abscesos múltiples son más controvertidos, y los mismos autores que antes recomendaban una actitud conservadora para los mismos, recomiendan actualmente una actitud más agresiva, puncionando al menos el de mayor tamaño, para obtener material para cultivo y aliviar la presión intracraneal ${ }^{15}$. Al margen de estos casos, el resto de los abscesos deben ser tratados quirúrgicamente, bien mediante punción del absceso, bien mediante cirugía abierta (craneotomía o craniectomía para vaciado del absceso o extirpación radical, incluyendo la cápsula).

El debate entre el drenaje del absceso, por un lado, y la cirugía abierta, por otro, es antiguo. Ya en 1926 Dandy se hacía eco de esta controversia, a favor del drenaje $\mathrm{p}^{7}$ El tratamiento mediante punción y drenaje del absceso presenta múltiples ventajas ${ }^{11}$ : permite con poca agresividad y anestesia local obtener material para cultivo y confirmación diagnóstica y alivia rápidamente la presión intracraneal; puede ser realizado en cualquier estadio de evolución del absceso; y la ayuda de la estereotaxia permite acceder a los abscesos más profundos o en áreas elocuentes del cerebro. Es considerado el tratamiento de elección por algunos autores ${ }^{26}$. La resección del absceso mediante cirugía abierta se recomienda para algunos tipos de abscesos, como los postraumáticos, dada la frecuencia con la que pueden retener cuerpos extraños ${ }^{11,19}$, los abscesos multiloculados, debido a la dificultad de su aspiración completa ${ }^{11,27}$, o los abscesos por hongos ${ }^{11}$. En los abscesos por nocardia hay controversias, y si bien algunos autores recomiendan la escisión, otros han demostrado su buena evolución mediante aspiración y tratamiento antibiótico, aunque debe recurrirse a la cirugía abierta sin dilación en caso de que no se demuestre radiológicamente buena respuesta en 2-4 semanas ${ }^{10,14}$. Young y Frazee recomiendan también la escisión para abscesos con gas en su interior ${ }^{29}$ : aunque el gas puede ser producido por gérmenes, con gran frecuencia se debe a una comunicación del parénquima cerebral con el exterior, y la búsqueda de dicha comunicación es una indicación de cirugía abierta. Respecto a los abscesos de cerebelo, aunque algunos autores prefieren tratarlos mediante aspiración $^{3}$, en nuestro Servicio son generalmente tratados mediante cirugía abierta, por el riesgo de deterioro súbito.

En nuestra serie, el tratamiento más utilizado ha sido la punción del absceso para aspiración del contenido purulento y el drenaje continuo a través de un catéter alojado en su cavidad durante unas 24-48 horas. Este tratamiento ha arrojado unos resultados excelentes el 90\% de los casos, con una estancia media de 13 días. La cirugía abierta ha sido el siguiente tratamiento realizado. En este grupo no sólo se incluye la resección capsular del absceso, sino también los casos en los que el absceso fue drenado mediante craneotomía, dejando la cápsula del absceso "in situ”. Este tratamiento ha obtenido unos resultados óptimos en nuestra 


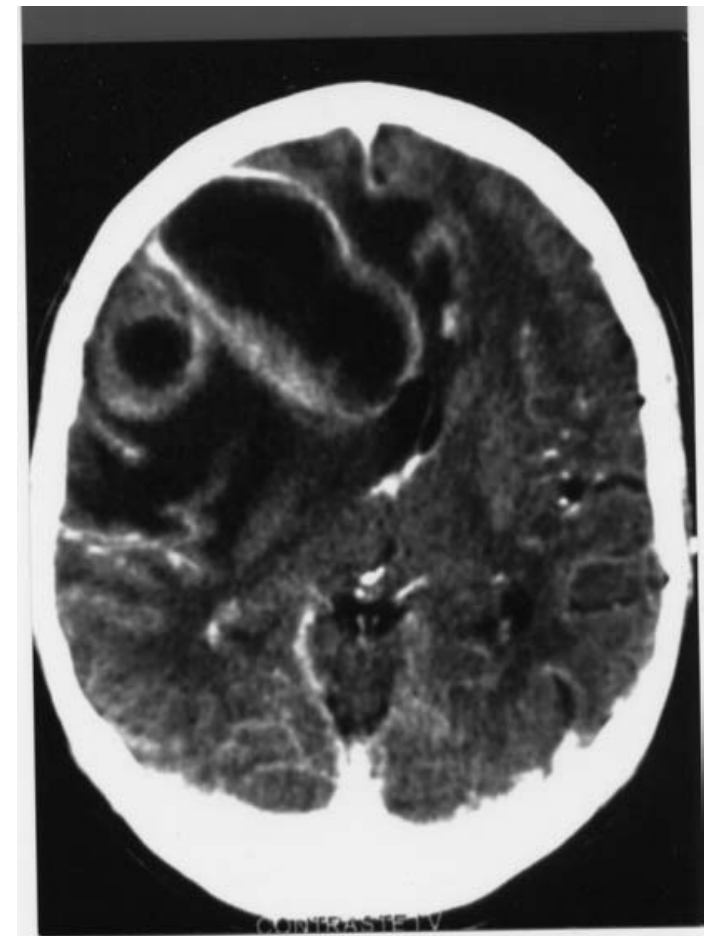

Figura 1 a. TC craneal con contraste que muestra un gran absceso frontal derecho, multiloculado, con importante desplazamiento de línea media, que fue tratado mediante punción y aspiración del contenido purulento a través de dos trépanos, dejando en la cavidad de mayor tamaño un catéter para drenaje continuo por gravedad.

serie en el $85 \%$ de los casos, con una estancia media de 26 días. Si bien los resultados en los casos de la aspiración del absceso son excelentes, hay que señalar que en 8 casos (un $22 \%$ ) hubo de realizarse una reintervención, que en 3 casos fue craneotomía, frente al $10 \%$ de reintervenciones en pacientes tratados con craneotomía como primera opción. El tratamiento exclusivamente médico sólo se utilizó en 5 pacientes $(8.3 \%)$ y en 4 de ellos hubo que recurrir ulteriormente a la punción-drenaje del absceso.

Las estancias medias merecen un comentario especial. Como hemos visto, se caracterizan por ser mucho mayores en pacientes tratados con craneotomía que en los tratados mediante drenaje del absceso (26 vs 13). Esta estancia hospitalaria se refiere a la estancia en nuestro Hospital, como centro de referencia de patología neuroquirúrgica. Muchos de estos pacientes completan su recuperación y el tratamiento antibiótico parenteral en su Hospital de Zona, lo que obviamente acorta las estancias en nuestro Servicio. No obstante, creemos importante reseñar la diferencia de estancias medias entre uno y otro tipo de tratamiento quirúrgico, pues una menor estancia facilita el uso de recursos especializados para nuevos requerimientos. La estancia media hospitalaria total, es decir, contabilizando la estancia
2006; 17: 23-33

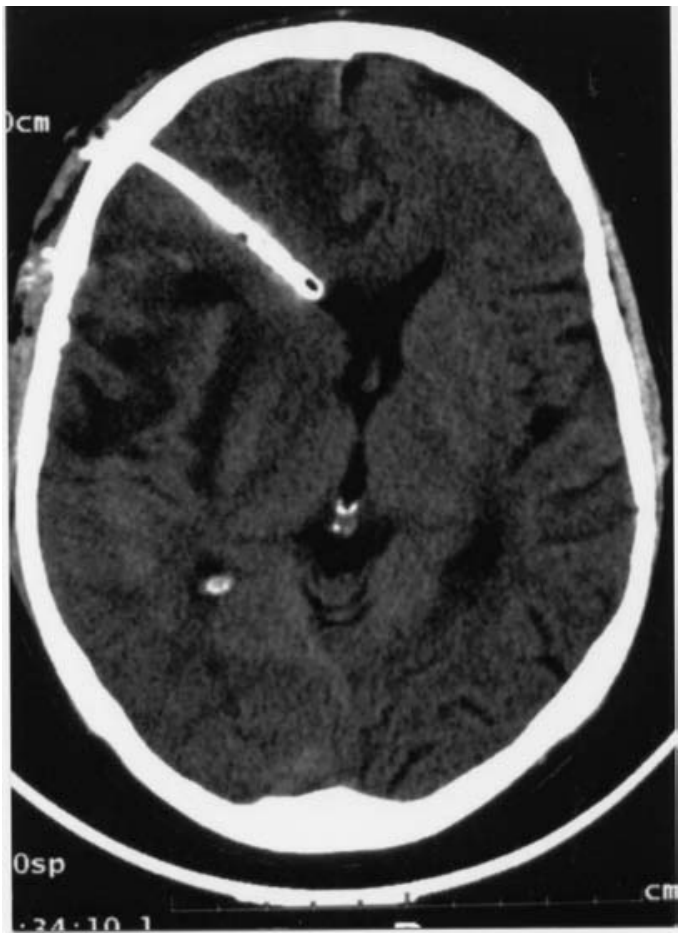

Figura 1 b. TC craneal del paciente anterior, realizado a las 24 horas de la intervención, en el que se aprecia la persistencia del catéter del drenaje, así como la desaparición del absceso y la mejoría del desplazamiento de la línea media.

en el hospital de referencia y el de zona, puede disminuirse mediante el empleo de los sistemas de hospitalización de día, que permitirán, en pacientes seleccionados, la aplicación ambulatoria de los tratamientos antibióticos parenterales $^{2}$.

El tratamiento se ha completado con el régimen de antibioterapia dictado por el Servicio de Enfermedades Infecciosas, corticoides y profilaxis anticomicial. Los corticoides, que hemos utilizado en un $75 \%$ de los pacientes, son un tratamiento controvertido. Se sabe que los esteroides restablecen la integridad de la barrera hematoencefálica, disminuyendo su permeabilidad, dando lugar a una rápida desaparición del edema cerebral, mejorando los déficits del paciente y su nivel de conciencia. Pero su propia actividad antiinflamatoria e inmunosupresora es la que los convierte en un tratamiento discutido en el caso de las infecciones cerebrales. Según pusieron de relieve Quartey y cols. en 1976, los corticoides dificultan el encapsulamiento de la infección y facilitan su diseminación ${ }^{20}$. Esta conclusión, no obstante, no fue ratificada plenamente por Schroeder y cols. en un trabajo posterior (1987), quienes apreciaron sólo un retraso en la encapsulación, sin afectar a la mortalidad ${ }^{25}$. Pese a ello, se aconseja restringir el uso de los esteroides a los casos de pacientes con incremento de 
la PIC con riesgo de enclavamiento y retirarlos tan pronto como sea posible ${ }^{15}$. Entre nosotros se han utilizado en un $75 \%$ de los pacientes, sin que parezcan haber influido de manera decisiva en el resultado final.

Se ha señalado repetidamente que el factor pronóstico de mortalidad más importante en los abscesos cerebrales es el nivel de conciencia al ingreso ${ }^{4,5,11,16,17,18}$. Otros factores pronósticos señalados, aunque discutidos, son: la fuente de la infección (peor los hematógenos por ser más frecuentemente múltiples y profundos $)^{5,11,21}$, el tamaño ${ }^{16}$, la edad por encima de los 40 años $^{2,5}$, el sexo femenino ${ }^{5}$, el número de $\operatorname{abscesos}^{18}$ y la velocidad de la progresión (peor los de instauración aguda $)^{5,9,11}$. En nuestra serie observamos una mortalidad mayor en pacientes con baja puntuación de Glasgow al ingreso, y especialmente en los pacientes de edad avanzada ( $>$ de 70 años).

\section{Conclusiones}

En nuestro trabajo hemos observado un alto nivel de eficacia de la punción del absceso, con una baja estancia hospitalaria. Pese a que esta modalidad de tratamiento requiere una reintervención en más ocasiones que la craneotomía, su escasa morbilidad, y su menor índice de estancias en un Servicio de alta especialización, como es el Servicio de Neurocirugía, justifican plenamente su uso como primera opción terapéutica siempre que no haya contraindicación expresa. La cirugía abierta constituye el adecuado complemento a este tipo de tratamiento y no se debe dudar en recurrir a ella cuando el drenaje no produce la mejoría esperada en un plazo razonable de tiempo.

\section{Bibliografía}

1. Blanco García, A., García Vázquez, E., Benito N., et al.: Absceso cerebral. Estudio clínico microbiológico y análisis pronóstico de 59 casos. Rev Clin Esp. 1998; 198: 413-419.

2. Borrás, J.M., García-Bach, M., Maestro-de León, J.I., et al: Colecciones purulentas intracraneales. Revisión de 34 casos tratados quirúrgicamente a lo largo de 12 años (19892000). Neurocirugía. 2002; 13: 6-14.

3. Brydon, H.L. and Hardwidge, C.: The management of cerebellar abscess since the introduction of CT scanning. Br J Neurosurg. 1994; 8: 447-455.

4. Ciurea, A.V., Stoica, F., Vasilescu, G., Nuteanu, L.: Neurosurgical management of brain abscesses in children. Child's Nery Syst. 1999; 15: 309-317.

5. González García, J., Gelabert, M., Pravos, A.G., Villa, J.M.F.: Colecciones purulentas intracraneales. Revisión de 100 casos. Rev Neurol. 1999; 29: 416-424.

6. Gormley, W.B., del Busto, R., Saravolatz, L.D., Rosenblum, M.L.: Cranial and intracranial bacterial infections. En Youmans (ed). Neurological Surgery. Philadelphia; W.B.
Saunders Company, 1996; pp 3191-3220.

7. Habib, A.A. and Mozaffar, T.: Brain abscess. Arch Neurol. 2001; 58: 1302-1304.

8. Heineman, H.S., Braude, Al, Osterholm, J.L.: Intracranial suppurative disease. Early presumptive diagnosis and successful treatment without surgery. JAMA 1971; 218: 15421547

9. Kao, P.T., Tseng, H.K., Liu, C.P., Su, S.C., Lee, C.M.: Brain abscess: clinical analysis of 53 cases. J Microbiol Inmunol Infect. 2003; 36: 129-136.

10. Lee, G.Y., Daniel, R.T., Brophy, B.P, Reily, P.L.: Surgical treatment of nocardial brain abscesses. Neurosurgery 2002; 51: 668-671.

11. Loftus, C.M., Osenbach, R.K., Biller, J.: Diagnosis and management of brain abscess. En Wilkins R.H. and Reengachary S.S.(eds). Neurosurgery. New York; Mc Graw-Hill, 1996; pp 3285-3298.

12. de Louvois, J., Gortavai, P., Hurley, R.: Bacteriology of abscesses of the central nervous system: a multicentre prospective study. Br Med J. 1977; 15: 981-984.

13. Macewen, W.: Pyogenic infective disease of the brain and spinal cord. New York; Macmillan and Co, 1893; pp 333334.

14. Mamelak, A.N., Obana, W.G., Flaherty, J.F., Rosemblum, M.L.: Nocardial brain abscess: treatment strategies and factors influencing outcome. Neurosurgery 1994; 35: 622631.

15. Mamelak, A.N., Mampalam, T.J., Obana, W.G., Rosemblum, M.L.: Improved management of multiple brain abscesses: a combined surgical and medical approach. Neurosurgery $1995 ; 36:$ 76-86.

16. Mampalam, T.J., Rosemblum, M.L.: Trends in the management of bacterial brain abscesses: a review of 102 cases over 17 years. Neurosurgery 1988; 23: 451-458.

17. Morgan, H., Wood, M.W., Murphey, F.: Experience with 88 consecutive cases of brain abscess. J Neurosurg. 1973; 38: 698-704.

18. Ortega, J.M., Díez Lobato, R., Aguado, J.M., Domínguez, J., Ayerbe, J., Sandoval, H., Rivas, J.J.: Absceso cerebral. Revisión de 60 casos. Neurocirugía 1992; 3: 125-130.

19. Patir, R., Sood, S., Bhatia, R.: Post-traumatic brain abscess: experience of 36 patients. Br J Neurosurg. 1995; 9: 29-35.

20. Quartey, G.R.C., Johnston, J.A., Rozdilsky, B.: Decadron in the treatment of cerebral abscess. J Neurosurg. 1976; 45: 301-310.

21. Qureshi, H.U., Habib, A.A., Siddiqui, A.A., Mozaffar, T., Sarwari, A.R.: Predictors of mortality in brain abscess. J Pak Med Assoc. 2002; 52: 111-116.

22. Roos, K.L.: Evaluation and treatment of patients with central nervous system infections. En Batjer \& Loftus (ed). Textbook of neurological surgery. Philadelphia; Williams \& Wilkins, 2003; pp 567-578. 
23. Rosemblum, M.L., Mampalam, T.J., Ponds, V.G.: Controversies in the management of brain abscess. Clin Neurosurg. 1986; 33: 603-632.

24. Rousseaux, M., Lesoin, F., Destee, A., Jomin, M., Petit, H.: Developments in the treatment and prognosis of multiple cerebral abscesses. Neurosurgery 1985; 16: 304-308.

25. Schroeder, K.A., McKeever, P.E., Schaberg, D.R., Hoff, J.T.: Effect of dexamethasone on experimental brain abscess. J Neurosurg. 1987; 66: 264-269.

26. Shahzadi, S., Lozano, A.M., Bernstein, M., Guha, A., Tasker, R.R.: Stereotactic management of bacterial brain abscesses. Can J Neurol Sci. 1996; 23: 34-39.

27. Su, T.M., Lan, C.M., Tsai, Y.D., Lee, T.C., Lu, C.H., Chang, W.N.: Multiloculated pyogenic brain abscess: experience with 25 patients. Neurosurgery 2003; 52: 1075 1080 .

\section{Comentario al trabajo: Abscesos piógenos encefálicos. Nuestra experiencia en 60 casos consecutivos de M. Ortega-Martínez y cols.}

En este trabajo de Ortega-Martínez y cols, los autores presentan su experiencia, analizando retrospectivamente 60 casos consecutivos de abscesos cerebrales atendidos en el Hospital Universitario Infanta Cristina de Badajoz durante 14 años. Analizan exhaustivamente las características clínicas y microbiológicas de los mismos, así como el manejo empleado. Esta patología, pese al gran descenso en su mortalidad que ha presentado en las últimas décadas, continúa representando una situación potencialmente muy grave que exige un adecuado manejo para su resolución.

Los autores preconizan como tratamiento quirúrgico de elección para los abscesos piógenos la punción aspiración, relegando la exéresis mediante craniotomía a circunstancias específicas. Esta es también la técnica empleada en la mayoría de los casos por nuestro Servicio después de la llegada de la $\mathrm{TC}^{2}$. La punción aspiración obtiene unos resultados prácticamente comparables a los de la craniotomía con una menor agresividad terapéutica.

Merece una mención especial lo referente a las pruebas diagnósticas de imagen. Los autores hacen hincapié en la TC craneal como la prueba radiológica fundamental. Ciertamente, la TC es el instrumento radiológico de elección para vigilar la evolución de los pacientes debido a su disponibilidad, sin embargo, el diagnóstico es con frecuencia
2006; 17: 23-33

28. Yang, S. and Zhao, C.: Review of 140 patients with brain abscess. Surg Neurol. 1993; 39: 290-296.

29. Young, R.F and Frazee, J.: Gas within intracranial abscess cavities: an indication for surgical excision. Ann Neurol. 1984; 16: 35-39.

Ortega-Martínez, M.; Cabezudo, J.M.; Fernández-Portales, I.; Gómez-Perals, L.; Rodríguez-Sánchez, J.A.; García-Yagüe, L.; Porras, L.F.; Lorenzana, L.M.; Ugarriza, F.; Pineda-Palomo, M.; Bernal-García, L.M.: Abscesos piógenos encefálicos. Nuestra experiencia en 60 casos consecutivos. Neurocirugía 2006; 17: 23-33.

Correspondencia postal: Dra. Marta Ortega Martínez. Servicio de Neurocirugía. Hospital Universitario Infanta Cristina. Avda de Elvas S/N. Badajoz.

dudoso con TC y es aquí donde la RM adquiere un papel preponderante. Esto es especialmente cierto hoy en día, debido a la llegada de nuevas secuencias que diagnostican con gran especificidad la presencia de abscesos. El diagnóstico diferencial radiológico entre tumor con necrosis central y absceso, que había resultado históricamente dificultoso, ha sufrido un giro con las nuevas secuencias de difusión, con las cuales se puede diferenciar el absceso (difusión disminuida) de la necrosis tumoral (difusión incrementada) con extraordinaria precisión ${ }^{1}$.

\section{Bibliografía}

1. Desprechins, B., Stadnik, T., Koerts, G., Shabana, W., Breucq, C., Osteaux, M.: Use of diffusion-weighted MR imaging in differential diagnosis between intracerebral necrotic tumors and cerebral abscesses. AJNR 1999; 20: 1252-1257.

2. Ortega, J.M., Lobato, R.D., Aguado, J.M., et al.: Absceso cerebral. Revisión de 60 casos. Neurocirugía 1992; 3: 125-130.

\section{Arrese \\ R.D. Lobato \\ Madrid}

